\title{
DISTRIBUTION OF ALTERNATION POINTS IN UNIFORM POLYNOMIAL APPROXIMATION
}

\author{
G. G. LORENTZ 1
}

\begin{abstract}
For a continuous function $f$ on $[0,1]$, we discuss the points where the polynomial $P_{n}(x)$ of best uniform approximation deviates most from $f(x)$, and the signs of the difference $f(x)-P_{n}(x)$ alternate. We show that these points can be very irregularly distributed in $[0,1]$, even if $f$ is entire.
\end{abstract}

1. Introduction. Problems discussed here concern uniform approximation of continuous functions by algebraic polynomials on $[0,1]$ and by trigonometric polynomials on the circle $T$.

By $Q_{n}:=Q_{n}(f)$ (also written $Q_{n}(x)$ ), we always denote the polynomial of degree $\leq n$ of best uniform approximation to $f \in C[0,1]$. By Chebyshev's theorem, if $f$ is not a polynomial, the $Q_{n}$ are characterized by the existence of a sequence of at least $n+2$ "alternation points" $x_{k}$,

$$
0 \leq x_{1}<\cdots<x_{N} \leq 1 \text {, }
$$

where $\left\|f-Q_{n}\right\|_{\infty}=\left|f(x)-Q_{n}(x)\right|$, and where the signs of $f(x)-Q_{n}(x)$ alternate:

$$
f\left(x_{k}\right)-Q_{n}\left(x_{k}\right)=(-1)^{k} \varepsilon\left\|f-Q_{n}\right\|, \quad \text { with } \varepsilon= \pm 1 .
$$

We denote by $N=N(n)$ the maximal length of an alternation sequence; then $N=n+2+s, s \geq 0$. This is equivalent to $Q_{n}=Q_{n+1}=\cdots=Q_{n+s} \neq Q_{n+s+1}$. By $A_{n}(f)$ we denote any set of alternation points (1.1) of $f$ of maximal length $N(n)$.

How are the alternation points (1.1) distributed in $[0,1]$ ? By a theorem of Kadec [4], for each $f$ there are infinitely many $n$ for which they are equidistributed. Let $d \mu=[x(1-x)]^{-1 / 2} d x$ be the Chebyshev measure on $[0,1]$. Reformulating Kadec's theorem, and slightly improving his estimate, we have

THEOREM 1. If $f \in C[0,1]$ is not a polynomial, then there exists an infinite increasing sequence of positive integers $\left\{n_{j}\right\}$ so that with $N_{j}=N\left(n_{j}\right)$, for each interval $I \subset[0,1]$,

$$
\left|A_{n_{j}}(f) \cap I\right|=\frac{\mu I}{\pi}\left(N_{j}+R_{j}\right), \quad\left|R_{j}\right| \leq C N_{j}^{1 / 2} \log N_{j}, \quad j=1,2, \ldots,
$$

where $C$ is an absolute constant.

(Kadec has $N_{j}^{(1 / 2)+\varepsilon}$ instead of $N_{j}^{1 / 2} \log N_{j}$.)

It is natural to ask whether equidistribution takes place for all large $n$ (instead of some large $n$ ), and if this is not true in general, whether it can be established at least when $f$ is nice, say analytic. The following theorem is a counterexample.

Received by the editors November 15, 1983. Presented to the American Mathematical Society, November 11, 1983, Evanston, Illinois.

1980 Mathematics Subject Classification. Primary 41A50, 42A10.

${ }^{1}$ Supported in part by NSF Grant \#MCS8303353. 
THEOREM 2. There exists an entire function $f$ and three infinite sequences of increasing positive integers $\left\{n_{j}^{(i)}\right\}, i=1,2,3$, so that for each $\varepsilon>0$, and all sufficiently large $j$,

$$
A_{n_{j}^{(1)}}(f) \subset[0, \varepsilon] ; \quad A_{n_{j}^{(2)}}(f) \subset[1-\varepsilon, 1],
$$

while $A_{n_{j}^{(3)}}$ is equidistributed in $[0,1]$ in the sense of (1.2).

This theorem holds also for trigonometric approximation-this is seen by the standard subtitution $x=$ cost.

We need some lemmas.

LEMMA 1 (FReUd; see [2, p. 82]). For each $f \in C[0,1]$, there is a constant $M=M(f)>0$ so that

$$
\|Q(g)-Q(f)\| \leq M\|g-f\|, \quad g \in C[0,1] .
$$

A polynomial $P_{n}$ of degree $\leq n$ is $\theta$-incomplete, $0<\theta<1$, if it has the form $P_{n}(x)=\sum_{[n \theta]}^{n} a_{k} x^{k}$. The following is a variant of a theorem of the author; see [5].

LEMMA 2. Let $0<\sigma<\theta^{2}$. Then for each $\varepsilon>0$ and each sufficiently large $n$, a $\theta$-incomplete polynomial $P_{n}$ with the property $\left|P_{n}(x)\right| \leq 1, x \in\left[\theta^{2}, 1\right]$ satisfies $\left|P_{n}(x)\right|<\varepsilon$ on $[0, \sigma]$.

Incomplete polynomials with special properties have been studied in detail by Saff and Varga [6]. They proved

LEMMA 3. For each $0<\theta<1$ and each $n$, there exists a $\theta$-incomplete polynomial $S_{n}$ with $\left\|S_{n}\right\|=1$ which attains $\geq n(1-\theta)$ maxima and minima on $\left[\theta^{2}, 1\right]$, with alternating values \pm 1 . 28]).

(The existence of such $S_{n}$ follows also from considerations of Bernstein [1, p.

2. Proof of the theorem. Let $\theta_{k} \rightarrow 1,0<\theta_{k}<1$. The coefficients $a_{j}>0$ will satisfy $\sum_{j=k}^{\infty} a_{j} \leq 2 a_{k}$ and, in addition, $a_{j+1}=o\left(a_{j}\right), j \rightarrow \infty$. We shall introduce another restriction for the $a_{j}$ later.

The integers $n_{k} \geq 1$ will be defined by induction and subject to the inequality

$$
n_{k} \leq\left(1-\theta_{k}\right) n_{k+1}-2 .
$$

If the $n_{1}, \ldots, n_{k}$ are known, we select $n_{k+1}$ so that (2.1) is satisfied, and, further, so that Lemma 2 applied to $S_{n_{k+1}}$ of Lemma 3 yields

$$
\left|S_{n_{k+1}}(x)\right|<k^{-1} \text { on }\left[0, \theta_{k}^{3}\right] \text {. }
$$

Then we define

$$
f(x)=\sum_{j=1}^{\infty} a_{j} \Pi_{j},
$$

where $\Pi_{j}(x)=S_{n_{j}}(x)$ for odd $j,=S_{n_{j}}(1-x)$ for even $j$. We let $f_{k}(x)=$ $\sum_{j=1}^{k+1} a_{j} \Pi_{j}$, and compare $Q_{n}=Q_{n}(f)$ and $Q_{n}^{*}:=Q_{n}\left(f_{k}\right)$, assuming that

$$
n_{k} \leq n<\left(1-\theta_{k}\right) n_{k+1}-2 \text {. }
$$


Let, for example, $k$ be even. We have $Q_{n}^{*}=\sum_{j=1}^{k} a_{j} \Pi_{j}$, since the sum is a polynomial of degree $\leq n$, and since $f_{k}-Q_{n}^{*}=a_{k+1} \Pi_{k+1}$ has in $\left[\theta_{k}^{2}, 1\right]$ an alternating sequence of length $\geq n+2$. We also see that for such $n,\left\|f_{k}-Q_{n}^{*}\right\|=\left|a_{k+1}\right|$. We further have

$$
\left\|f-f_{k}\right\| \leq \sum_{k+2}^{\infty} a_{j} \leq 2 a_{k+2}
$$

hence by Lemma 1 ,

$$
\left\|Q_{n}-Q_{n}^{*}\right\| \leq 2 M a_{k+2}
$$

We write

$$
f-Q_{n}=\left(f-f_{k}\right)+\left(f_{k}-Q_{n}^{*}\right)+\left(Q_{n}^{*}-Q_{n}\right)=a_{k+1} \Pi_{k+1}+h,
$$

and have $\|h\| \leq 2(M+1) a_{k+2}$. This shows that $\left\|f-Q_{n}\right\|=a_{k+1}(1+o(1))$. On the other hand, on $\left[0, \theta_{k}^{3}\right]$ we can use $(2.2)$ and get

$$
\left|f(x)-Q_{n}(x)\right| \leq 2(M+1) a_{k+2}+k^{-1} a_{k+1}=o\left(a_{k+1}\right) .
$$

Thus, all the alternation points of $f$, where $\left|f(x)-Q_{n}(x)\right|=\left\|f-Q_{n}\right\|$, lie in $\left[\theta_{k}^{3}, 1\right]$. And for odd $k$, they are contained in $\left[0,\left(1-\theta_{k}\right)^{3}\right]$.

To assure that $f$ is entire, we have to take very small $a_{j}$. If $M_{j}$ is the maximum of $\left|S_{n_{j}}(z)\right|$ on the disk $|z| \leq j$, it is sufficient to take $\sum_{1}^{\infty} a_{j} M_{j}<+\infty$. Finally, the third sequence $n_{j}^{(3)}$ exists by Theorem 1 .

3. Remarks. In spite of our main theorem one could still ask under what conditions one has equidistribution

$$
\left|A_{n}(f) \cap I\right|=(\mu I / \pi)(N+o(1))
$$

for all $n \rightarrow \infty$. We can show that a sufficient condition is a modest but steady decrease to zero of the degree of approximation, $E_{n}(f):=\min _{P_{n}}\left\|f-P_{n}\right\|$ :

$$
E_{n+1}(f) \leq\left(1-\varepsilon_{n}\right) E_{n}(f), \quad \text { with } \log \left(1 / \varepsilon_{n}\right)=o(n) .
$$

Unfortunately, one does not know what structural properties of $f$ ensure (3.2). It is true that the interesting paper of Fiedler and Jurkat [3] contains results of this type, but for the $L_{1}$-instead of the uniform-approximation.

\section{REFERENCES}

1. S. N. Bernstein, Leçons sur les propriétés extrémales et la meilleure approximation des fonctions analytiques d'une variable réelle, Gauthier-Villars, Paris, 1926.

2. E. W. Cheney, Introduction to approximation theory, McGraw-Hill, New York, 1966.

3. H. Fiedler and W. B. Jurkat, Best $L^{1}$-approximation by polymomials, J. Approximation Theory 37 (1983), 269-292.

4. M. I. Kadec, On the distribution of points of maximal deviation in the approximation of continuous functions by polymomials, Uspehi Mat. Nauk 15 (1960), 199-202.

5. J. H. B. Kemperman and G. G. Lorentz, Bounds for polynomials with applications, Indag. Math. 41 (1979), 13-26.

6. E. B. Saff and R. S. Varga, On incomplete polymomials, Numerische Methoden der Approximationstheorie (L. Collatz et al., eds.), Internat. Schriftenreihe Numer. Math., no. 42, Birkhäuser Verlag, Basel, 1978, pp. 281-298.

Department of Mathematics, University of Texas at Austin, Austin, Texas 78712 (Current address)

Department of Mathematics, University of Alberta, Edmonton, Canada T6G 2G1 